\title{
An Efficient Perfectly Matched Layer Based Multilevel Fast Multipole Algorithm for Large Planar Microwave Structures
}

\author{
Dries Vande Ginste, Eric Michielssen, Fellow, IEEE, Femke Olyslager, Fellow, IEEE, and \\ Daniël De Zutter, Fellow, IEEE
}

\begin{abstract}
An efficient multilevel fast multipole algorithm (MLFMA) formalism to model radiation and scattering by/from large planar microwave structures is presented. The technique relies on an electric field integral equation (EFIE) formulation and a series expansion for the Green dyadic, based on the use of perfectly matched layers (PML). In this way, a new PML-MLFMA is developed to efficiently evaluate matrix-vector multiplications arising in the iterative solution of the scattering problem. The computational complexity of the new algorithm scales down to $O(N)$ for electrically large structures. The theory is validated by means of several illustrative, numerical examples.
\end{abstract}

Index Terms-Microstrip structure, multilevel fast multipole algorithm (MLFMA), perfectly matched layer (PML), planar antenna array.

\section{INTRODUCTION}

$\mathbf{M}$ ANY full-wave microwave and MMIC planar circuit simulators rely on method of moments (MoM) [1] based integral equation solvers. These schemes use so-called Green functions to store all the characteristics of the layered medium. Only the system's metallic conductors are discretized into $N$ segments in order to determine their currents. The $N \times N$ linear system that arises from the MoM can be solved by LU-decomposition or by an iterative method [2]. Often one uses an electrical field integral equation (EFIE) to model the field problem and this will also be the approach adopted here. Nevertheless, the presented method is equally applicable for a mixed potential formulation. Unfortunately, the calculation of the elements of the Green dyadic $\overline{\mathbf{G}}_{e e}$ [3], [4] unavoidably calls for the time-consuming evaluation of Sommerfeld-type integrals [5]. Building the dense moment matrix is also computationally expensive. Recently, several methods have been proposed to reduce the computational complexity associated with the iterative solution of the linear systems, e.g. the adaptive integral method (AIM) [6], the thin-stratified medium fast-

Manuscript received December 9, 2004; revised October 29, 2005. The work of D. Vande Ginste was supported by a doctoral grant from the Flemish Institute for the Promotion of Scientific and Technological Research in the Industry (IWT).

D. Vande Ginste, F. Olyslager, and D. De Zutter are with the Department of Information Technology, Ghent University, Sint-Pietersnieuwstraat 41, 9000 Gent, Belgium (e-mail: dries.vande.ginste@intec.UGent.be).

E. Michielssen is with the Radiation Laboratory, Department of Electrical Engineering and Computer Science, University of Michigan, Ann Arbor, MI 48109 USA.

Digital Object Identifier 10.1109/TAP.2006.874320 multipole algorithm (TSM-FMA) [7], the fast inhomogeneous plane wave algorithm (FIPWA) [8]-[10] and the fast far-field approximation (FAFFA) [11].

A very efficient and elegant way to calculate Green functions, based on the use of perfectly matched layers (PML) [12]-[14], has been proposed in [15]-[17]. For a layered structure that is closed by a perfect electric conductor (PEC) plane at the top and bottom of the structure, the Sommerfeld integrals that arise in the Green function calculations can be expressed as a series of surface waves. By using a PML that is covered by a PEC plane, one can also close open layered media while maintaining the open character of the structure. In [15], [16] this approach was used to obtain an analytic and easy to determine series representation for the Green functions of open layered media.

In this paper, the PML-MoM formalism is combined with a fast multipole method (FMM) [18]-[20] in order to model the scattering from planar metallizations on a microstrip substrate. This problem has been studied for several years (see, e.g., [21]-[25]). Our new procedure has already proven its efficiency in two dimensions, i.e., for a set of parallel microstrip lines [26], [27]. In this contribution the two-dimensional technique is extended to a multilevel fast multipole algorithm (MLFMA) [28]-[31] for arbitrary planar metallizations, allowing us to store the linear system with low memory requirements and to solve it fast and efficiently. Classical iterative methods have a computational complexity of order $O\left(N^{2}\right)$ to perform one matrix-vector multiplication. Since the separate terms in the series representation of the Green dyadic can be decomposed into a set of plane waves with controllable accuracy, an operation count of $O(N)$ is achieved. It may be clear that the applications for radiation by large planar microstrip structures are numerous, e.g. the modeling of large printed antenna arrays.

The FIPWA technique presented in [8] for two-dimensional (2-D) scattering and its extensions to three dimensions (3-D) [9] is also based on a series expansion for the Green function. In the FIPWA technique each Sommerfeld integral is replaced by a properly chosen steepest descent path integral, a constant phase branch cut integral and discrete surface wave pole contributions. The remaining integrals are efficiently discretized using Gaussian quadrature rules. Although this scheme, when applied to structures considered in this paper, also achieves an $O(N)$ complexity, our PML based series representation provides a valuable alternative to this technique. It avoids the usual steepest descent path complications when branch-points and/or 


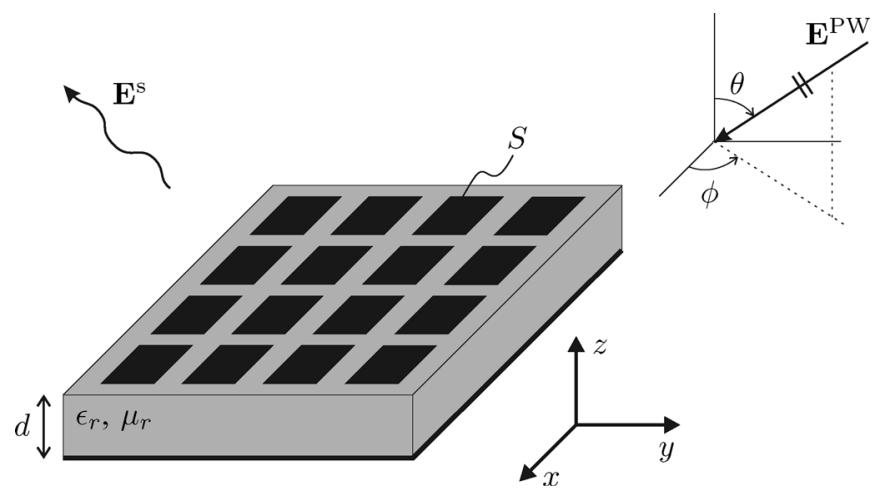

Fig. 1. Geometry.

surface wave poles come close to each other and start to interfere, leading to a more robust scheme. The PML-series comes in a natural way and can be calculated efficiently.

The method is illustrated by considering the radiation or the scattering by/from microstrip substrates covered by an arbitrary planar metallization. Nevertheless, it will be briefly shown that the presented technique is immediately applicable to general multilayered structures with planar and nonplanar metallizations on different levels. The theory of the new formalism is outlined in Section II. In Section III several illustrative, numerical examples validate the proposed technique.

Notation: all sources and fields are assumed time-harmonic with angular frequency $\omega$ and the time dependencies $e^{j \omega t}$ are suppressed.

\section{Formulation OF THE New TeChNiQue}

\section{A. Geometry and Classical Integral Equation Analysis}

Consider a microstrip structure comprising PEC planar elements $S$ (traces and/or patches) that reside in the $z=d$ plane on top of an infinite PEC-backed substrate of thickness $d$, permittivity $\epsilon_{1}=\epsilon_{0} \epsilon_{r}$, and permeability $\mu_{1}=\mu_{0} \mu_{r}$ (Fig. 1); here $\epsilon_{0}$ and $\mu_{0}$ denote the permittivity and permeability of the air-filled half-space $z>d$, and $\epsilon_{r}$ and $\mu_{r}$ are the relative permittivity and permeability of the substrate. In case of a lossy substrate, $\epsilon_{r}$ and $\mu_{r}$ are complex numbers. The incident electric field $\mathbf{E}^{\mathrm{i}}(\mathbf{r}=x \hat{\mathbf{x}}+y \hat{\mathbf{y}}+z \hat{\mathbf{z}})$, produced by impressed sources that radiate in the presence of the PEC-backed substrate, illuminates $S$. In response, the scattered electric field $\mathbf{E}^{\mathrm{S}}(\mathbf{r})$ is generated by electric surface currents $\breve{\mathbf{J}}(\boldsymbol{\rho}=x \hat{\boldsymbol{x}}+y \hat{\boldsymbol{y}})=$ $J_{x}(\boldsymbol{\rho}) \hat{\mathbf{x}}+J_{y}(\boldsymbol{\rho}) \hat{\mathbf{y}}$ on $S$. The total electric field $\mathbf{E}^{\mathrm{t}}(\mathbf{r})$ comprises both the incident and scattered fields: $\mathbf{E}^{\mathrm{t}}(\mathbf{r})=\mathbf{E}^{\mathrm{i}}(\mathbf{r})+\mathbf{E}^{\mathrm{s}}(\mathbf{r})$. Below, transverse to $z$ restrictions of $\mathbf{E}^{\mathrm{q}}(\boldsymbol{\rho}), \mathrm{q} \in\{\mathrm{i}, \mathrm{s}, \mathrm{t}\}$ are denoted $-\hat{\mathbf{z}} \times\left[\hat{\mathbf{z}} \times \mathbf{E}^{\mathrm{q}}(\boldsymbol{\rho})\right] \equiv \check{\mathbf{E}}^{\mathrm{q}}(\boldsymbol{\rho})=E_{x}^{\mathrm{q}}(\boldsymbol{\rho}) \hat{\mathbf{x}}+E_{y}^{\mathrm{q}}(\boldsymbol{\rho}) \hat{\mathbf{y}}$.

An electric field integral equation (EFIE) for $\breve{\mathbf{J}}(\boldsymbol{\rho})$ is constructed by enforcing the component of $\check{\mathbf{E}}^{\mathrm{t}}(\boldsymbol{\rho})$ tangential to $S$ to vanish. By expressing transverse to $z$ electric fields in the $z=d$ plane produced by $\breve{\mathbf{J}}(\boldsymbol{\rho})$ using the transverse electric-current-toelectric-field Green dyadic $\overline{\mathbf{G}}_{e e}\left(\boldsymbol{\rho} \mid \boldsymbol{\rho}^{\prime}\right)$, which is detailed in Section II-B, the boundary condition $\breve{\mathbf{E}}^{\mathrm{t}}(\boldsymbol{\rho})=\check{\mathbf{E}}^{\mathrm{i}}(\boldsymbol{\rho})+\check{\mathbf{E}}^{\mathrm{S}}(\boldsymbol{\rho})=$ $\mathbf{0}, \forall \boldsymbol{\rho} \in S$ is cast as

$$
-\check{\mathbf{E}}^{\mathrm{i}}(\boldsymbol{\rho})=\check{\mathbf{E}}^{\mathrm{S}}(\boldsymbol{\rho}) \equiv \iint_{S} \overline{\mathbf{G}}_{e e}\left(\boldsymbol{\rho} \mid \boldsymbol{\rho}^{\prime}\right) \cdot \check{\mathbf{J}}\left(\boldsymbol{\rho}^{\prime}\right) d \boldsymbol{\rho}^{\prime}, \quad \forall \boldsymbol{\rho} \in S .
$$
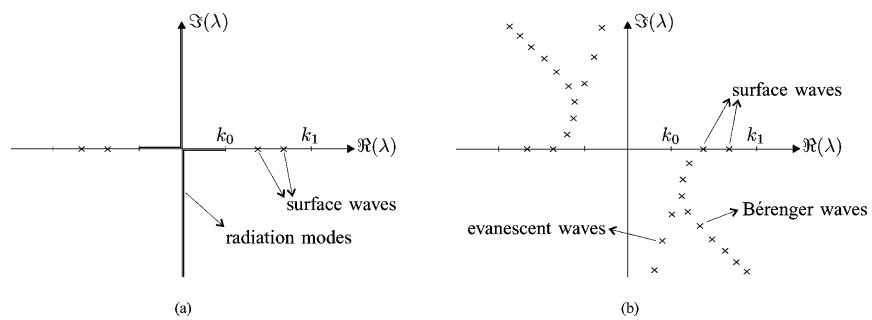

Fig. 2. Modal spectrum. (a) Open substrate and (b) PML-closed substrate.

The integral (1) is solved by the MoM [1]. To this end, $S$ is approximated by a (potentially nonuniform) rectilinear mesh with $N$ interior edges. Next, $\breve{\mathbf{J}}(\boldsymbol{\rho})$ is expanded in a set of vector rooftop basis functions $\check{\mathbf{w}}_{j}(\boldsymbol{\rho})=w_{x, j}(\boldsymbol{\rho}) \hat{\mathbf{x}}+w_{y, j}(\boldsymbol{\rho}) \hat{\mathbf{y}}, j=$ $1, \ldots, N$ [32] with support $S_{j} \subset S$ comprising two patches that are joined by the mesh's $j$ th interior edge as

$$
\check{\mathbf{J}}(\boldsymbol{\rho})=\sum_{j=1}^{N} I_{j} \check{\mathbf{w}}_{j}(\boldsymbol{\rho})
$$

Inserting (2) into (1) and applying a Galerkin testing procedure yields the following $N \times N$ linear system in the unknown expansion coefficients $I_{j}, j=1, \ldots, N$ :

$$
\mathbf{V}=\overline{\mathbf{Z}} \cdot \mathbf{I}
$$

The $N$-vector I contains the expansion coefficients $I_{j}, j=$ $1, \ldots, N$ and the elements of the $N$-vector $\mathbf{V}$ and the $N \times N$ matrix $\overline{\mathbf{Z}}$ are given by

$$
\begin{aligned}
V_{i} & =-\iint_{S_{i}} \check{\mathbf{E}}^{\mathrm{i}}(\boldsymbol{\rho}) \cdot \check{\mathbf{w}}_{i}(\boldsymbol{\rho}) d \boldsymbol{\rho} \\
Z_{i j} & =\iint_{S_{i}} \iint_{S_{j}} \check{\mathbf{w}}_{i}(\boldsymbol{\rho}) \cdot \overline{\mathbf{G}}_{e e}\left(\boldsymbol{\rho} \mid \boldsymbol{\rho}^{\prime}\right) \cdot \check{\mathbf{w}}_{j}\left(\boldsymbol{\rho}^{\prime}\right) d \boldsymbol{\rho}^{\prime} d \rho .
\end{aligned}
$$

Linear system (3) can be solved using direct or iterative schemes. Iterative solution schemes, e.g. the BiConjugate Gradients stabilized method (BiCGstab) [2], are amenable to acceleration by fast multipole methods [33] (and their descendants) as they only require the multiplication of the moment matrix $\overline{\mathbf{Z}}$ by a test vector $\mathbf{I}$; hence, they are adopted here.

\section{B. Green Dyadic With the Use of a PML}

The modal spectrum of the open microstrip substrate of Fig. 1 comprises some discrete propagating surface waves and a continuous set of radiation modes [34], [35]. Provided the substrate is lossless, these surface waves' transverse modal propagation constants $\lambda$ can be found on the real axis between the $k_{0}$ and $k_{1}=k_{0} \sqrt{\epsilon_{r} \mu_{r}}$. Here, $k_{0}=\omega / c$ is the free space wavenumber and $c$ is the speed of light. The radiation modes are characterized by propagation constants $\lambda$ located along a branch cut [see Fig. 2(a)]. For lossy materials, the propagation constants of the surface waves in the right half plane will shift downwards in the complex plane. Of course, all the modes come in pairs with propagation constants of opposite sign. The branch cut causes the numerical evaluation of Sommerfeld-integrals [5], [35] and this complicates the application of the MLFMA to the acceleration of matrix-vector products (3). 


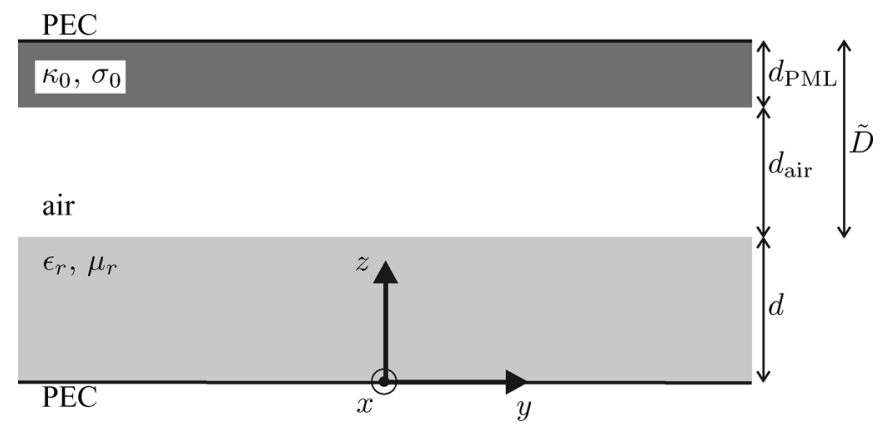

Fig. 3. PML-closed substrate.

The crux of our new formalism is based on the application of PMLs [12]-[14]. The PML-concept is invoked to obtain an approximate analytical/closed-form expression for $\overline{\mathbf{G}}_{e e}\left(\boldsymbol{\rho} \mid \boldsymbol{\rho}^{\prime}\right)$ involving only discrete modes. To this end, the air-filled half-space above the microstrip substrate of Fig. 1 is closed by putting a PML, with material parameters $\kappa_{0}$ and $\sigma_{0}$, backed by a PEC on top of it, as is shown in Fig. 3. Previous investigations have shown that, by complex coordinate stretching, the air-PML combination can be treated as one single air layer with complex thickness $\tilde{D}=d_{\mathrm{air}}+d_{\mathrm{PML}}\left(\kappa_{0}-j\left(\sigma_{0} / \omega \epsilon_{0}\right)\right)=\Gamma e^{-\zeta}$ [13], [15], [16], [36], [37]. The PML-closed substrate mimics the behavior of the original, open substrate. The original modal spectrum is now replaced by a discrete set of modes of the PML-closed substrate [see Fig. 2(b)] [17]. The propagation constants $\lambda_{\mathrm{TM}, n}$ and $\lambda_{\mathrm{TE}, n}, n=1, \ldots, \infty$, of this structure's TM- and TE-modes satisfy their corresponding dispersion relations of the PEC-dielectric-air-PML-PEC structure

$$
\text { for } \lambda_{\mathrm{TX}, n}: \quad Y_{1}^{\mathrm{TX}} \cot \left(\gamma_{1} d\right)+Y_{0}^{\mathrm{TX}} \cot \left(\gamma_{0} \tilde{D}\right)=0
$$

where here, and in what follows, TX stands for TM and TE, and with

$$
\begin{aligned}
Y_{m}^{\mathrm{TM}} & =\frac{j \omega \epsilon_{m}}{\gamma_{m}} \\
Y_{m}^{\mathrm{TE}} & =\frac{\gamma_{m}}{j \omega \mu_{m}} \\
\gamma_{m} & =\sqrt{\omega^{2} \epsilon_{m} \mu_{m}-\lambda^{2}}
\end{aligned}
$$

and $m=0,1$. Since the propagation constants are defined only up to a sign, only those with $\Re(\lambda)>0$ are retained when solving (6). Both the TM- and the TE-modes come in three flavors. First, there are the propagating surface waves that are (virtually) identical to those found in the original open microstrip substrate. Next, there are the evanescent or pseudo-leaky surface waves. And finally, there are the so-called Bérenger surface waves [38]. Below, no distinction between these three types of modes is made and they are collectively indexed such that $\left|\Im\left(\lambda_{\mathrm{TX}, n}\right)\right|<\left|\Im\left(\lambda_{\mathrm{TX}, n+1}\right)\right|$ for all $n$.

By applying a spectral domain technique [5] and using Cauchy's residue theorem for the inverse Hankel transform, the Green dyadic for a horizontal dipole source at $\boldsymbol{\rho}^{\prime}+d \hat{\mathbf{z}}$ can be expressed as a series of TM- and TE-modes of the closed waveguide. Introducing the transverse to $z$ nabla operator $\check{\nabla}=(\partial / \partial x) \hat{\mathbf{x}}+(\partial / \partial y) \hat{\mathbf{y}}$ yields the following equation for $\overline{\mathbf{G}}_{e e}\left(\rho \mid \boldsymbol{\rho}^{\prime}\right)[16]:$

$$
\begin{aligned}
\overline{\mathbf{G}}_{e e}\left(\boldsymbol{\rho} \mid \boldsymbol{\rho}^{\prime}\right) \approx & \frac{1}{2 \omega} \sum_{n} \frac{1}{\lambda_{\mathrm{TM}, n}^{2} M^{\mathrm{TM}}\left(\lambda_{\mathrm{TM}, n}\right)} \check{\nabla} \check{\nabla} H_{0}^{(2)} \\
& \times\left(\lambda_{\mathrm{TM}, n}\left|\boldsymbol{\rho}-\boldsymbol{\rho}^{\prime}\right|\right) \\
& +\frac{\omega}{2} \sum_{n} \frac{1}{\lambda_{\mathrm{TE}, n}^{2} M^{\mathrm{TE}}\left(\lambda_{\mathrm{TE}, n}\right)}(\hat{\mathbf{z}} \times \check{\nabla})(\hat{\mathbf{z}} \times \check{\nabla}) \\
& \times H_{0}^{(2)}\left(\lambda_{\mathrm{TE}, n}\left|\boldsymbol{\rho}-\boldsymbol{\rho}^{\prime}\right|\right)
\end{aligned}
$$

where dyadic notation was used [39]. Here, $H_{0}^{(2)}(\cdot)$ is the zeroth-order Hankel function of the second kind and

$$
\begin{aligned}
M^{\mathrm{TM}}(\lambda)= & \frac{\epsilon_{1} \cot \gamma_{1} d}{\gamma_{1}^{3}}+\frac{\epsilon_{1} d}{\gamma_{1}^{2} \sin ^{2} \gamma_{1} d}+\frac{\epsilon_{0} \cot \gamma_{0} \tilde{D}}{\gamma_{0}^{3}} \\
& +\frac{\epsilon_{0} \tilde{D}}{\gamma_{0}^{2} \sin ^{2} \gamma_{0} \tilde{D}} \\
M^{\mathrm{TE}}(\lambda)= & \frac{d}{\mu_{1}} \frac{1}{\sin ^{2} \gamma_{1} d}-\frac{\cot \gamma_{1} d}{\mu_{1} \gamma_{1}}+\frac{\tilde{D}}{\mu_{0}} \frac{1}{\sin ^{2} \gamma_{0} \tilde{D}} \\
& -\frac{\cot \gamma_{0} \tilde{D}}{\mu_{0} \gamma_{0}} .
\end{aligned}
$$

The summations in (10) extend all TM- or all TE-modes $(n=$ $1, \ldots, \infty)$.

Several remarks regarding the above series expansion of $\overline{\mathbf{G}}_{e e}\left(\boldsymbol{\rho} \mid \boldsymbol{\rho}^{\prime}\right)$ that are important in its intended application are in order.

- The Hankel kernel in (10) can be seen as the Green function of a 2-D Helmholtz equation in a homogeneous space. Hence, by applying the PML-concept the Green function of the original open microstrip problem in three dimensions is decomposed into a set of two-dimensional homogeneous space Green functions. This decomposition will play a crucial role in the construction of the new MLFMA (see Section II-D).

- Proofs of the completeness of the modes of a PML-closed structure are given in [40], [41]. Of course, in practical applications one cannot use an infinite number of modes. However, only a limited number of modes is needed owing to the fact that the propagation constants of the higher order modes have large negative imaginary parts. Even more, for a certain accuracy of $\overline{\mathbf{G}}_{e e}$ the number of modes needed in (10) decreases rapidly with the distance $\left|\boldsymbol{\rho}-\boldsymbol{\rho}^{\prime}\right|$.

- Close to the source point the series representation becomes impractical [17], [42]. This means that for small $\left|\boldsymbol{\rho}-\boldsymbol{\rho}^{\prime}\right|$ and in particular for the self-patch interactions in the MoM, a classical technique for evaluating the Green operator remains in order. Because of our intended goal, viz. the use of series representation (10) in an MLFMA framework, this restriction poses no problem, as the MLFMA itself requires near-fields to be evaluated separately from far-fields [33]. 


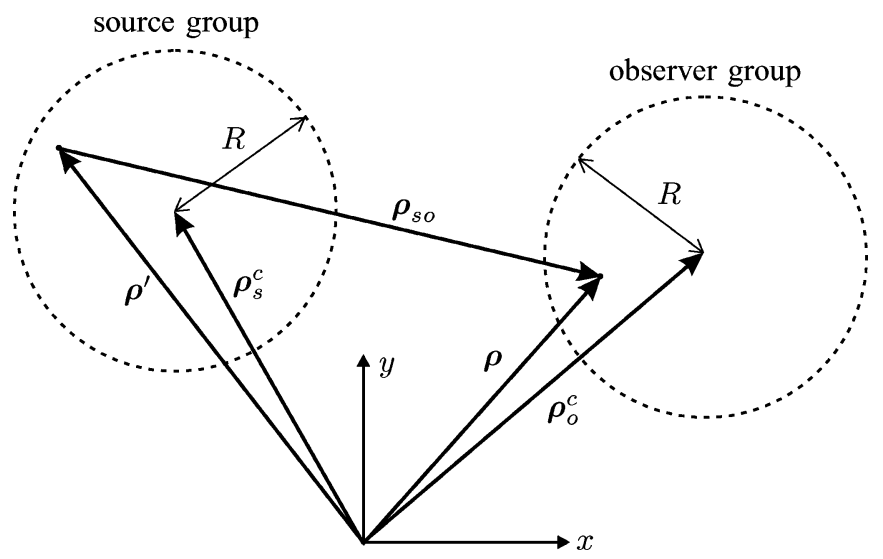

Fig. 4. Constellation of source and observer.

\section{Plane Wave Decomposition of the Hankel Kernel}

Helmholtz equation FMMs [18]-[20] rely on so-called diagonal translation operators, viz. expansions of the pertinent Green functions in terms of plane waves, to represent fields produced by distributed sources at sufficiently separated observers. Here, our focus is on a plane wave decomposition of the kernel of the Green dyadic (10), viz. the Hankel function $H_{0}^{(2)}\left(\lambda\left|\boldsymbol{\rho}-\boldsymbol{\rho}^{\prime}\right|\right)$. Consider the configuration of Fig. 4, comprising a source point at $\rho^{\prime}$ and an observer point at $\rho$ that belong to source and observer constellations contained in circles of radius $R$ centered about $\boldsymbol{\rho}_{s}^{c}$ and $\boldsymbol{\rho}_{o}^{c}$. It was shown in [33], [43] that the kernel can be expressed as:

$$
\begin{aligned}
& H_{0}^{(2)}\left(\lambda\left|\boldsymbol{\rho}-\boldsymbol{\rho}^{\prime}\right|\right) \approx \sum_{q=-Q}^{Q} e^{j \check{\boldsymbol{\lambda}}\left(\phi_{q}\right) \cdot\left(\boldsymbol{\rho}^{\prime}-\boldsymbol{\rho}_{s}^{c}\right)} T_{q}\left(\lambda,\left|\boldsymbol{\rho}_{s o}^{c c}\right|, \phi_{s o}^{c c}\right) \\
& \times e^{-j \check{\lambda}\left(\phi_{q}\right) \cdot\left(\boldsymbol{\rho}-\boldsymbol{\rho}_{o}^{c}\right)} \\
& =\sum_{q=-Q}^{Q} \mathrm{PW}_{q} \\
& T_{q}(\lambda, \rho, \phi)=\frac{1}{2 Q+1} \sum_{q^{\prime}=-Q}^{Q} H_{q^{\prime}}^{(2)}(\lambda \rho) e^{j q^{\prime}\left(\phi-\phi_{q}-\frac{\pi}{2}\right)}
\end{aligned}
$$

with $\boldsymbol{\rho}_{s o}^{c c}=\boldsymbol{\rho}_{o}^{c}-\boldsymbol{\rho}_{s}^{c}, \phi_{s o}^{c c}=\arctan \left(\left(\hat{\mathbf{x}} \cdot \boldsymbol{\rho}_{s o}^{c c}\right) /\left(\hat{\mathbf{y}} \cdot \boldsymbol{\rho}_{s o}^{c c}\right)\right)$ and $\check{\boldsymbol{\lambda}}(\phi)=\lambda(\cos \phi \hat{\mathbf{x}}+\sin \phi \hat{\mathbf{y}})$. Equation (13) realizes a plane wave decomposition of the Hankel function. Its physical interpretation is illuminating. The radiation pattern of the source group is sampled into $2 Q+1$ outgoing plane waves referenced w.r.t. the center of the source group and traveling in directions $\phi_{q}=2 q \pi /(2 Q+1), q=-Q, \ldots, Q$. Upon multiplication by the translation operator (14) the outgoing plane waves are converted into $2 Q+1$ incoming plane waves referenced w.r.t. the center of the observation group. Then the contribution of each plane wave is measured at the observer. Expansion (13) is only valid when the source group and the observer group are well-separated, meaning e.g. $\left|\boldsymbol{\rho}_{s o}^{c c}\right|>5 R$. If this condition is satisfied and if $\lambda$ is real, then $2 Q+1$, viz. the number of plane waves participating in the expansion, can be chosen as

$$
2 Q+1=4 \lambda R+C(\lambda R)^{1 / 3}
$$

where $C$ is a constant that sets the MLFMA accuracy level [33]. Note, however, that the wavenumbers in (10) are complex wavenumbers; therefore, the above (classical) estimate (15) does not apply. Hence, in our case, $Q$ will be determined using a simple search procedure, as will be outlined in Section II-D.

\section{A MLFMA for 3-D Microstrip Structures}

Let us now combine the results from the Sections II-B and II-C. Consider a dipole source with strength $\check{\boldsymbol{\alpha}}=\alpha_{x} \hat{\mathbf{x}}+\alpha_{y} \hat{\mathbf{y}}$ located on the substrate-air interface at position $\mathbf{r}^{\prime}=\boldsymbol{\rho}^{\prime}+d \hat{\mathbf{z}}$. By using (1), it is easy to see that the field radiated by this source at an observer placed on the substrate at position $\mathbf{r}=\boldsymbol{\rho}+d \hat{\mathbf{z}}$ is given by

$$
\check{\mathbf{E}}(\boldsymbol{\rho})=\overline{\mathbf{G}}_{e e}\left(\boldsymbol{\rho} \mid \boldsymbol{\rho}^{\prime}\right) \cdot \check{\boldsymbol{\alpha}}
$$

For the Green dyadic in (16) the PML-series (10) is used and each Hankel kernel in this series will be substituted by its plane wave decomposition (13). Before doing so the unit vectors

$$
\begin{aligned}
\hat{\mathbf{n}}_{q} & =\cos \phi_{q} \hat{\mathbf{x}}+\sin \phi_{q} \hat{\mathbf{y}} \\
\hat{\mathbf{t}}_{q} & =-\sin \phi_{q} \hat{\mathbf{x}}+\cos \phi_{q} \hat{\mathbf{y}}
\end{aligned}
$$

are introduced and the following identities are derived from (13)

$$
\begin{aligned}
& \check{\boldsymbol{\nabla}} \check{\nabla} \cdot H_{0}^{(2)}\left(\lambda\left|\boldsymbol{\rho}-\boldsymbol{\rho}^{\prime}\right|\right) \check{\boldsymbol{\alpha}} \\
& \approx-\lambda^{2} \sum_{q=-Q}^{Q} \operatorname{PW}_{q} \hat{\mathbf{n}}_{q} \hat{\mathbf{n}}_{q} \cdot \check{\boldsymbol{\alpha}} \\
& (\hat{\mathbf{z}} \times \check{\nabla})(\hat{\mathbf{z}} \times \check{\nabla}) \cdot H_{0}^{(2)}\left(\lambda\left|\boldsymbol{\rho}-\boldsymbol{\rho}^{\prime}\right|\right) \check{\boldsymbol{\alpha}} \\
& \approx-\lambda^{2} \sum_{q=-Q}^{Q} \mathrm{PW}_{q} \hat{\mathbf{t}}_{q} \hat{\mathbf{t}}_{q} \cdot \check{\boldsymbol{\alpha}} .
\end{aligned}
$$

Making the above described substitutions now and also using (19) and (20), yields the following expression:

$$
\begin{aligned}
\check{\mathbf{E}}(\boldsymbol{\rho}) \approx & \frac{1}{2 \omega} \sum_{n} \frac{1}{M^{\mathrm{TM}}\left(\lambda_{\mathrm{TM}, n}\right)} \sum_{q=-Q_{\mathrm{TM}, n}}^{Q_{\mathrm{TM}, n}} \mathrm{PW}_{q} \hat{\mathbf{n}}_{q} \hat{\mathbf{n}}_{q} \cdot \check{\boldsymbol{\alpha}} \\
& +\frac{\omega}{2} \sum_{n} \frac{1}{M^{\mathrm{TE}}\left(\lambda_{\mathrm{TE}, n}\right)} \sum_{q=-Q_{\mathrm{TE}, n}}^{Q_{\mathrm{TE}, n}} \mathrm{PW}_{q} \hat{\mathbf{t}}_{q} \hat{\mathbf{t}}_{q} \cdot \check{\boldsymbol{\alpha}}
\end{aligned}
$$

where subscripts on $Q_{\mathrm{TX}, n}$ indicate that the number $Q$ in (13) depends on the propagation constant $\lambda_{\mathrm{TX}, n}$. Similar subscripts on $\mathrm{PW}_{q}, \hat{\mathbf{n}}_{q}$ and $\hat{\mathbf{t}}_{q}$ have been omitted for simplicity. This equation reflects the fact that, when calculating the field radiated by an arbitrarily oriented dipole in the $z=d$ metallization plane, only longitudinal and transverse components of the plane wave decomposed electric fields are to be retained when considering TM- and TE-polarized fields respectively. Equation (21) is the core formula of our new PML-MLFMA for microstrip structures.

It is noted that the original MLFMAs were conceived for computing fields produced by known source constellations residing in lossless media. When applying the method to the computation of fields in lossy media, care should be exercised when sampling and translating far-field signatures to account for their 
excess bandwidth and avoid loss of accuracy. In practice, this often can be achieved through the use of renormalized far-field signatures (defined on multiple phase centers per box) in conjunction with windowed translation operators. Needless to say, these techniques also could be applied in the present context to improve the quality of the MLFMA approximation for any mode characterized by a complex transverse wavenumber. In practice, we chose to not implement them and use MLFMA techniques designed for lossless media because for all practical substrates considered, MLFMA inaccuracies stemming from the imaginary transverse wavenumbers of the higher order modes are very small compared to the field from the fundamental mode (see Section III). Moreover, if somehow this condition would be violated, the mode in question always can be accounted for classically, viz. without using the MLFMA, as its field vanishes beyond the (quasi-)near-field.

The reader is encouraged to consult references [28]-[31] to gain familiarity with the basic MLFMA scheme for free space environments. In what follows, only those details of an " $N N_{l}$-level MLFMA" pertinent to its adoption into the above described integral equation solver, are presented.

\section{1) Geometrical subdivision of $S$}

In the proposed $N_{l}$-level scheme, $S$ is enclosed in a hypothetical square box of side length $D$ that is partitioned recursively $N_{l}-1$ times to create a uniform quad-tree. The number of levels is chosen such that the side length of the smallest box thus obtained is about a fifth of the free-space wavelength $\lambda_{0}=2 \pi / k_{0}$. The large box containing $S$ is said to reside at level $N_{l}$; the smallest boxes are said to reside on level 1. Specifically, starting at the highest (coarsest) level, each level $l+1$ "parent" box is subdivided into four equal size level $l$ "child" boxes of circumscribing radius $R_{l}$. A basis function is said to belong to a box if its center of mass resides in that box. Furthermore, a box is said to be empty if it contains no basis functions. In the process of subdividing boxes, empty boxes are immediately discarded. Next, at all levels and starting with the highest one, all far-field box pairs are identified. A pair of boxes constitutes a far-field pair if (i) the distance between their closest points is greater than a fixed constant, typically chosen as $5 R_{l}$ - see the above restriction on the use of the MLFMA - and (ii) their parent boxes do not constitute a far-field pair. At the lowest (densest) level, all box pairs (including self-pairs) not identified as far-field pairs are labeled near-field pairs.

2) Classical evaluation of interactions

Contributions to the right-hand side of (5) due to spatial basis functions contained in near-field pairs are evaluated classically, that is, without using MLFMA and PML concepts.

\section{3) MLFMA evaluation of interactions}

Interactions between basis functions belonging to far-field pairs are computed using the proposed PMLMLFMA scheme. That is, all rooftop basis and testing functions are approximated by a set of dipoles with locations and weights determined by a Gaussian quadrature rule, and interactions between dipoles are computed using (21).

\section{4) Mode trimming}

In the final remarks of Section II-B it is stated that for a certain accuracy of the PML-series for $\overline{\mathbf{G}}_{e e}$ (10) the number of modes decreases rapidly with the distance $\left|\boldsymbol{\rho}-\boldsymbol{\rho}^{\prime}\right|$ between a source and an observer. Therefore, for the calculation of interactions that take place at high levels in the MLMFA tree-and hence for large distances $\left|\boldsymbol{\rho}-\boldsymbol{\rho}^{\prime}\right|$-one can use less modes than at low levels without destroying the accuracy. Let $M_{\mathrm{TX}, l}$ denote the number of TX PML-modes used at a certain level $l$ in the tree. For increasing level number $l, M_{\mathrm{TX}, l}$ decreases substantially. This will also be shown numerically in Section III-A. An important consequence is that the cost of the algorithm is not linearly dependent on the number of modes. This is a major improvement w.r.t. to our previous 2-D implementation [27] and this new feature is named mode trimming.

\section{5) Computational complexity}

By using the PML-paradigm, the 3-D layered medium problem can be seen as a set of 2-D scattering problems in free space; each element of the set corresponds to one PML-mode and thus, to one Hankel function. For these kind of 2-D scattering problems an MLFMA results in a reduction in computational complexity from $O\left(N^{2}\right)$ to $O(N \log N)$ and $O(N)$ for surface-bound and volumetric source constellations respectively (see [33]). Contrary to the 2-D implementation of the PML-FMM [27], using $M$ modes in the 3-D PML-MLFMA algorithm does not mean now that the computational complexity scales as $O(M N \log N)$ or $O(M N)$. Because of the mode trimming property described above, this linear dependency with $M$ can be omitted. Hence, if $S$ represents dense metallizations, e.g. a planar microstrip array, the complexity of the scheme scales as $O(N)$. In the worst case scenario, when $S$ represents sparse metallization, e.g. a long microstrip trace, the complexity scales as $O(N \log N)$.

\section{6) Determination of the sampling rates $2 Q_{\mathrm{TX}, n}+1$}

To determine the minimum sampling rate $2 Q_{\mathrm{TX}, n}+1$, a simple search procedure is used to ensure a given level of accuracy of expansion (21) by requiring that

$\left|\frac{H_{0}^{(2)}\left(\lambda_{\mathrm{TX}, n}\left|\rho-\boldsymbol{\rho}^{\prime}\right|\right)-\sum_{q=-Q_{\mathrm{TX}, n}}^{Q_{\mathrm{TX}, n}} \mathrm{PW}_{q}}{H_{0}^{(2)}\left(\lambda_{\mathrm{TM}, 1} \rho_{\min }\right)}\right|<\xi$

where $\xi$ is the desired accuracy for (21) and $\rho_{\min }$ is the minimal distance between two groups placed far enough from each other to use the decomposition. The propagation constant $\lambda_{\mathrm{TM}, 1}$ belongs to the fundamental TM-surface wave which is always supported by the microstrip substrates under consideration. Given the loci of the modes retained in (21) this method typically restricts $2 Q_{\mathrm{TX}, n}+1$ to a number equal to, or smaller than, that needed to represent the fundamental surface wave by itself. Enforcement of a given level of relative accuracy for each mode separately would increase the number of plane waves for the highly evanescent modes to impractical levels and/or nonconvergence of the series 
(21). Using (22) will lead to very accurate results for the total algorithm without the increase of the sampling rates and hence, without destroying the computational complexity (see Section III-A).

Remark: The above theory can easily be extended to general multilayered structures with planar and nonplanar metallizations on different levels. Without going into much detail, the gist of this extension is explained here.

In every layer $i$ of the structure-determined by the material parameters $\epsilon_{i}$ and $\mu_{i}$-the $z$-dependency of the currents needs to be included. Of course, this requires an adaptation of the Green functions. It is easy to show (by application of the spectral domain technique [5] and the PML-paradigm) that schematically, in each layer $i$, the following PML-series is obtained:

$$
G_{i}\left(\rho, z \mid z^{\prime}\right) \approx \sum_{n} c_{n, i} H_{0}^{(2)}\left(\lambda_{\mathrm{TX}, n} \rho\right) e^{ \pm j \gamma_{\mathrm{TX}, n, i}\left(z-z^{\prime}\right)}
$$

with

$$
\gamma_{\mathrm{TX}, n, i}=\sqrt{\omega^{2} \epsilon_{i} \mu_{i}-\lambda_{\mathrm{TX}, n}^{2}}
$$

and where $c_{n, i}$ is a constant. Hence, in every layer $i$ the (transversal to $z$ )-dependency has not been modified. For each $i$ the $z$-dependency can be written as an exponential-like function (i.e. a sine, a cosine, etc) that only depends on $z^{\prime}$ and $z$, i.e., the $z$-coordinates of the source and the observer respectively, and on the propagation constant $\lambda_{\mathrm{TX}, n}$ of the PML-modes. These transverse wavenumbers are solutions of the TX-polarized dispersion relations of the multilayered PML-waveguide. To implement an MLFMA, the source and observer contributions have to separated. The Hankel kernel can still be written as its plane wave decomposition. The exponential functions can be factorized immediately.

For completeness it is also mentioned here that it is perfectly possible to introduce a second PEC-backed PML at $z=-\tilde{D}$. This has to be done in case the (multilayered) substrate is not backed by a ground plane.

\section{NUMERICAL RESULTS}

This section comprises three subsections. First, the correctness of the new method will be demonstrated numerically and it will also be shown that the sampling rates in the plane wave decomposition (13) do not increase for higher order modes. Second, the high computational efficiency of our formalism will be demonstrated, in comparison with a classical technique. Third, some illustrative examples are given. Emphasis is on showing the reader that the proposed method is indeed suited for a variety of large 3-D microstrip problems. All simulations were carried out on a Linuxbased 2.4 GHz Pentium IV PC with 2 GB RAM and a BiCGstab iterative solver was used to solve the linear systems (3).

\section{A. Validation of the Method}

The accuracy of our PML-MLFMA code is controlled by many parameters. Several of them were described in Section II (e.g., PML parameters $\Gamma, \zeta$, and MLFMA parameters $Q_{\mathrm{TM}, n}$, $Q_{\mathrm{TE}, n}, M_{\mathrm{TM}, l}, M_{\mathrm{TE}, l}$, and $\xi$ ). Unfortunately, within the limited scope of this paper it is not possible to introduce and discuss all of them; this is especially true for parameters linked to

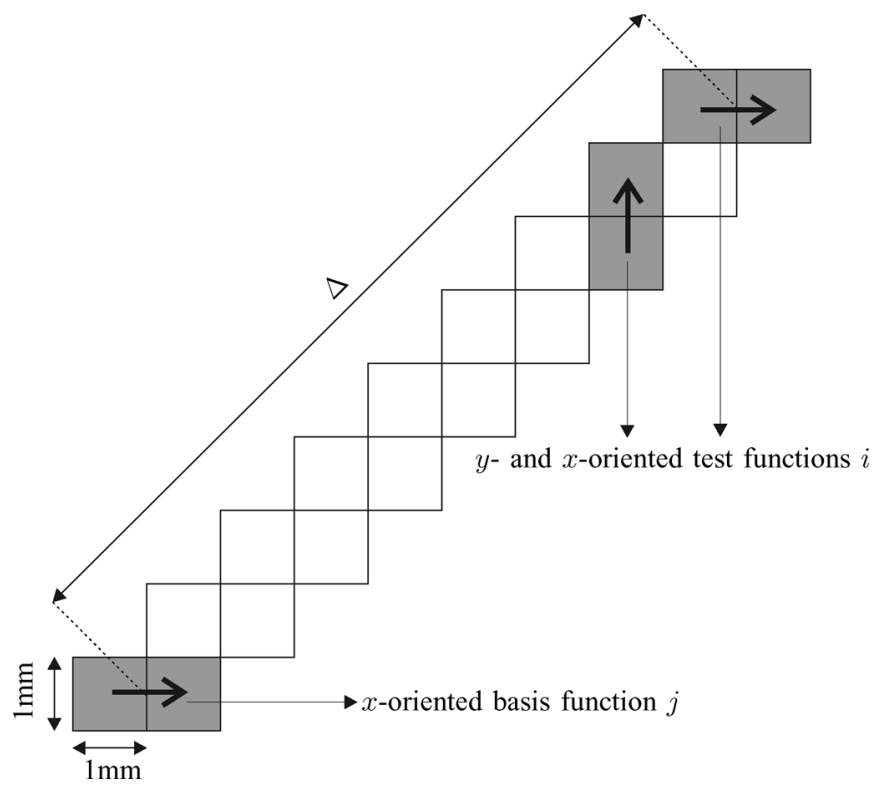

Fig. 5. Layout of the metallization used for testing the accuracy of the method.

the MLFMA component of our code (e.g., various oversampling factors, spatial quadratures, etc.). One of the input parameters to our PML-MLFMA code is the target accuracy $\mathcal{E}$, defined as the average relative error of the far-field matrix elements computed by using the PML-MLFMA paradigm. Upon specification of this parameter, all code parameters adjust to a critical value that guarantees this target accuracy without wasting computational resources; parameter selection is achieved either by (approximate) analytic means $\left(M_{\mathrm{TM}, l}, M_{\mathrm{TE}, l}, \xi, \Gamma\right.$, and $\zeta$ see [16], [17], [27]), or by brute-force local searches $\left(Q_{\mathrm{TM}, n}\right.$ and $\left.Q_{\mathrm{TE}, n}\right)$.

To verify the usefulness of the PML-MLFMA for modeling a microstrip geometry, consider the metallization depicted in Fig. 5. This metallization is separated from a PEC ground plane by an air-substrate with $\epsilon_{r}=\mu_{r}=1$ of thickness $d=1 \mathrm{~mm}$. The angular frequency is $\omega=2 \pi 10 \cdot 10^{9} \mathrm{~Hz}$. This air-substrate is chosen for two reasons. First, for this configuration, the transverse Green dyadic is known analytically

$$
\begin{aligned}
\overline{\mathbf{G}}_{e e}\left(\boldsymbol{\rho} \mid \boldsymbol{\rho}^{\prime}\right)= & \frac{1}{j \omega \epsilon_{0} 4 \pi}\left[\check{\nabla} \check{\nabla}+k_{0}^{2} \overline{\mathbf{I}}\right] \\
& \times\left(\frac{e^{-j k_{0}\left|\boldsymbol{\rho}-\boldsymbol{\rho}^{\prime}\right|}}{\left|\boldsymbol{\rho}-\boldsymbol{\rho}^{\prime}\right|}-\frac{e^{-j k_{0} \sqrt{\left|\boldsymbol{\rho}-\boldsymbol{\rho}^{\prime}\right|^{2}+4 d^{2}}}}{\sqrt{\left|\boldsymbol{\rho}-\boldsymbol{\rho}^{\prime}\right|^{2}+4 d^{2}}}\right)
\end{aligned}
$$

where $\overline{\mathbf{I}}$ is the two-dimensional identity dyadic. The moment matrix elements $Z_{i j}^{\text {class }}$, calculated by evaluating (5) using (25), will be used as a very precise reference in order to check the accuracy of the new method. Second, for this configuration, the transverse wavenumbers of the pertinent PML-waveguide, viz. an air-filled parallel plate waveguide of complex thickness $d+$ $\tilde{D}$, are also analytically known

$$
\lambda_{\mathrm{TM}, n}=\lambda_{\mathrm{TE}, n}=\sqrt{k_{0}^{2}-\left(\frac{n \pi}{d+\tilde{D}}\right)^{2}}, \quad n=1, \ldots, \infty .
$$

For other substrates one needs to solve the dispersion relations (6) numerically. Note that while this PML-waveguide also supports a TEM-mode, it is never excited as the currents on $S$ flow transverse 


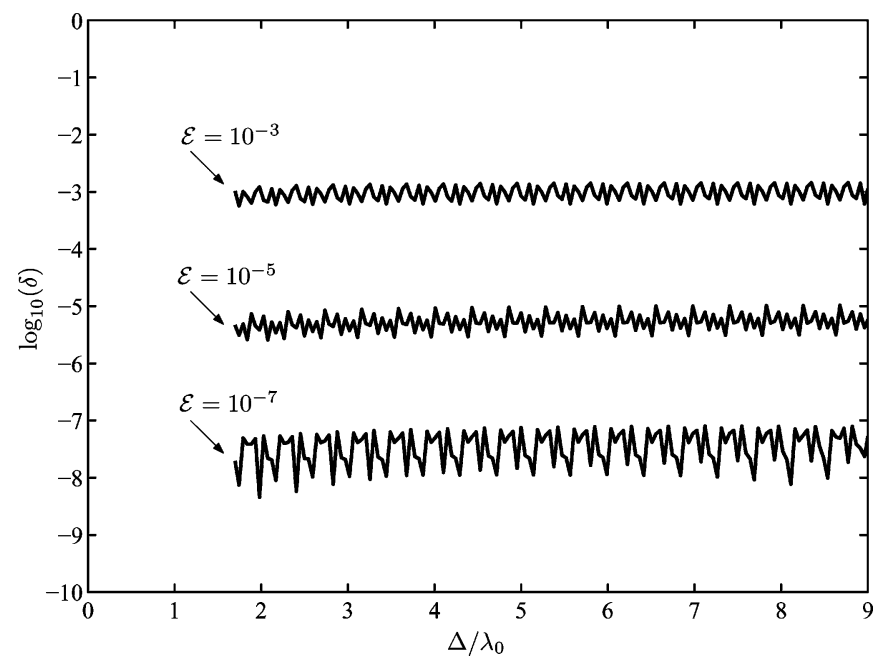

Fig. 6. Relative error $\delta$ on the $x-x$-interactions as a function of the distance $\Delta$ and for different values of $\mathcal{E}$.

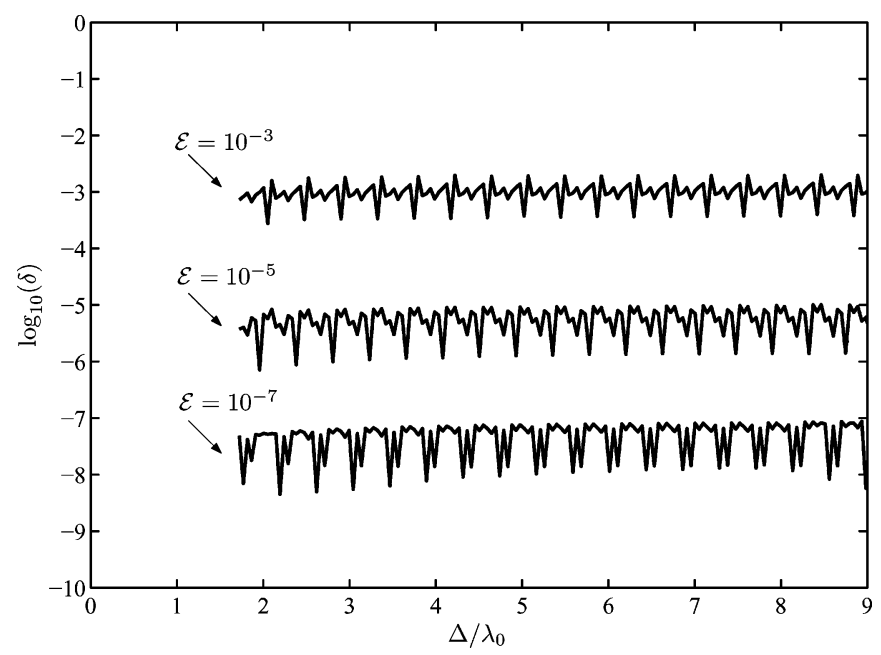

Fig. 7. Relative error $\delta$ on the $x-y$-interactions as a function of the distance $\Delta$ and for different values of $\mathcal{E}$.

to $z$. Name $Z_{i j}^{\mathrm{MLFMA}}$ an element of the moment matrix of our new method, supposing that all interactions are far and thus by making use of the PML and MLFMA. The elements $Z_{i j}^{\mathrm{MLFMA}}$ are evaluated by consecutively multiplying the moment matrix with test vectors equal to the columns of the unit matrix.

Let us now compare $Z_{i j}^{\text {class }}$ with $Z_{i j}^{\mathrm{MLFMA}}$. For an increasing distance $\Delta$, as indicated in Fig. 5 , between the basis function and the test function, viz. for an increasing $|i-j|$, the relative error $\delta(\Delta)$ is given by

$$
\delta(\Delta)=\left|\frac{Z_{i j}^{\text {class }}-Z_{i j}^{\mathrm{MLFMA}}}{Z_{i j}^{\text {class }}}\right| .
$$

Due to the staircase layout of the metallization, one is able to evaluate the interaction between a basis and a test function that have the a parallel or an orthogonal orientation, allowing to check all four elements of the Green dyadic. The accuracy of the method is shown in Figs. 6 and 7 for a varying target accuracy $\mathcal{E}$. Fig. 6 gives the results for the $x-x$-interactions, i.e., for the matrix elements describing interactions between $x$-oriented basis and test functions. In Fig. 7 the results for $x-y$-interactions, viz. for the matrix elements describing interactions between
TABLE I

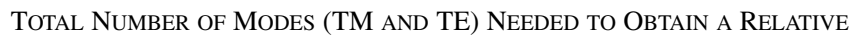
ERROR OF $10^{-6}$ AND $10^{-4}$ ON THE $x-x$-INTERACTIONS GIVEN A CERTAin Distance $\Delta$

\begin{tabular}{|c|c||c|c|}
\hline \multicolumn{2}{|c||}{ Distance } & \multicolumn{2}{c|}{ Total number of modes needed } \\
$\Delta[\mathrm{mm}]$ & $\Delta / \lambda_{0}$ & $\delta=10^{-6}$ & $\delta=10^{-4}$ \\
\hline 70.71 & 2.359 & 32 & 22 \\
106.1 & 3.538 & 24 & 18 \\
141.4 & 4.717 & 20 & 16 \\
176.8 & 5.897 & 16 & 12 \\
212.1 & 7.076 & 12 & 10 \\
\hline
\end{tabular}

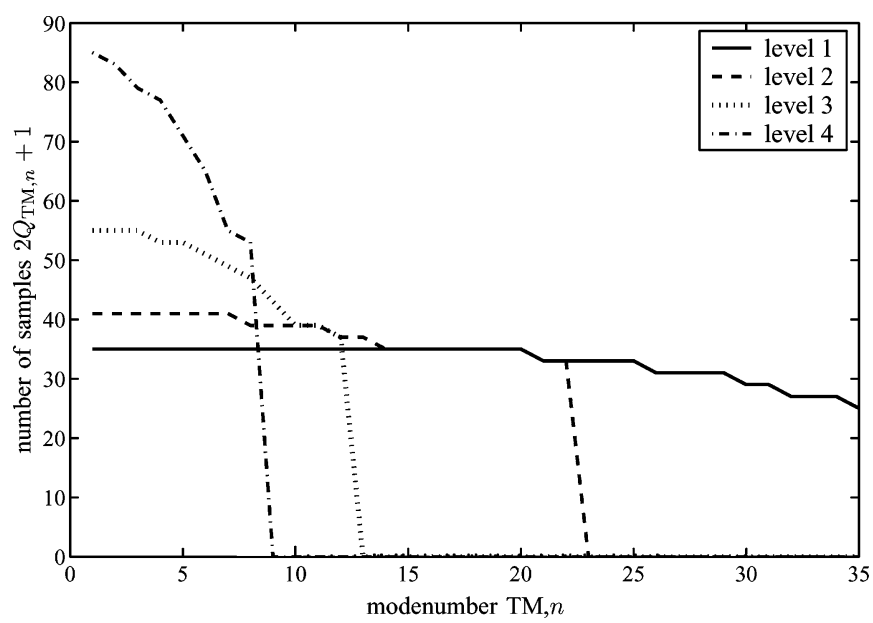

Fig. 8. Number of samples at four levels needed for each TM-mode to obtain an accuracy of $10^{-7}$.

$x$-oriented basis functions and $y$-oriented test functions or vice versa, are shown. The reader notices that the target accuracies $\mathcal{E}$ can easily be reached. The radius of the groups at the lowest level is $R_{1}=0.3 \lambda_{0}$. This might seem quite small (often one uses $0.5 \lambda_{0}$ or even $\lambda_{0}$ ), but as one notices, it does not cause accuracy problems. Even better, the MLFMA can be used starting from small distances (here $\Delta=51 \mathrm{~mm}$, which corresponds to a distance of $5 R_{1}$ between a pair of boxes at level 1 that constitute a far-field pair), and hence take full advantage of the technique. Below this distance, a classical technique needs to be adopted. In the example here, four levels in our MLFMA tree are used.

In Section II-D the concept of mode trimming is introduced, meaning that the number of modes $M_{\mathrm{TM}, l}$ and $M_{\mathrm{TE}, l}$ decreases with increasing level number $l$. An increasing $l$ means of course that the distance $\Delta$ between a basis and a test function increases. In Table I it can be seen that it is perfectly safe to trim the modes without loss of accuracy. In the table we aimed at an accuracy of $\mathcal{E}=10^{-6}$ and $\mathcal{E}=10^{-4}$ for the $x-x$-interactions. In Fig. 8 the sampling rates $2 Q_{\mathrm{TM}, n}+1$ are plotted for the TM-modes at four levels, for a target accuracy $\mathcal{E}=10^{-7}$, as used in the example of Figs. 6 and 7. When the number of samples drops to zero, this means of course that the mode is not used at that particular level as is requested by the mode trimming feature. Also, the sampling rate does not increase for higher order modes, on the contrary. This indicates that one can use the relative error for the core MLFMA (21) as defined in (22). It also means that the plane wave decomposition for complex wavenumbers $\lambda_{\mathrm{TX}, n}, \mathrm{TX}=$ 


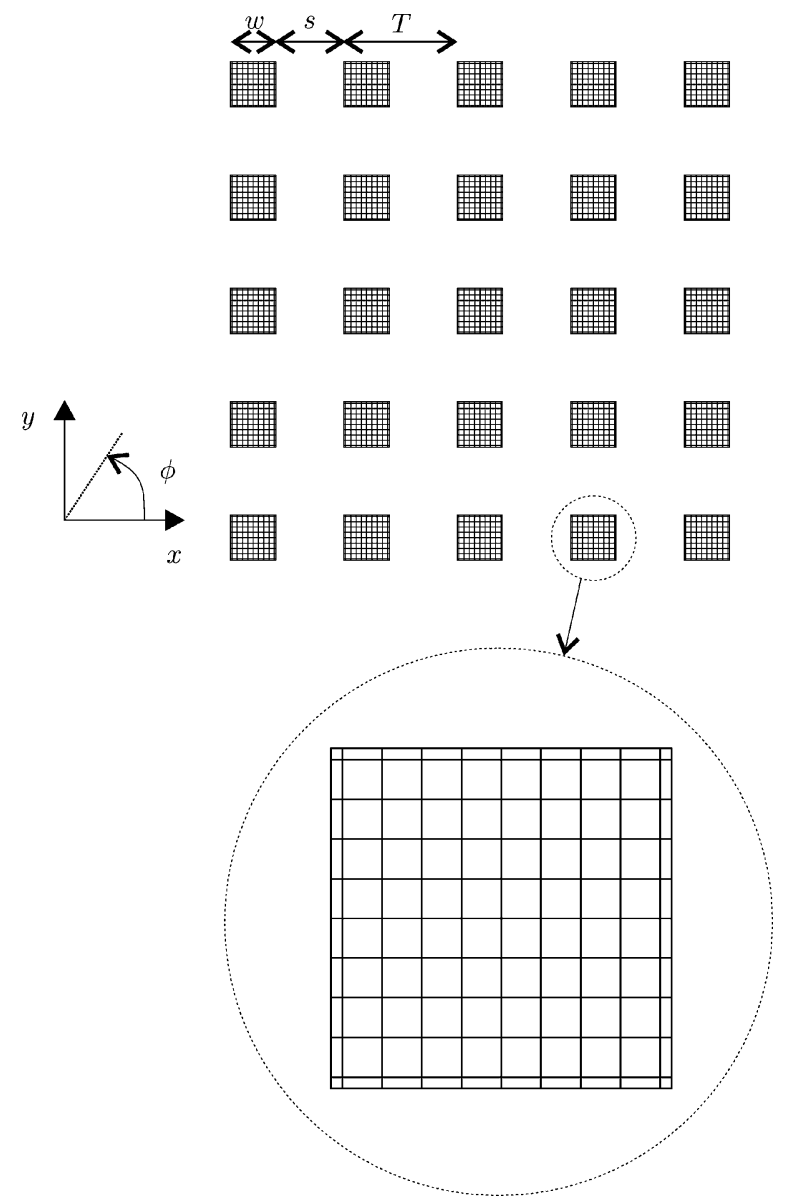

Fig. 9. Layout of the metallization used for testing the computational complexity of the method.

TE or TM, does not destroy the computational complexity of the algorithm. Remark that in the case of a microstrip substrate, with $k_{1} \neq k_{0}$, the substrate does propagate surface waves (with wavenumbers $\lambda \in \mathbb{R}$ ). In this case the mode trimming is even more efficient, since these modes will more and more dominate the total accuracy as the distance increases.

One can conclude that with the new method a fully controllable accuracy is achieved.

\section{B. Computational Efficiency}

For testing the computational complexity of our new MLFMA, a substrate with thickness $d=3.17 \mathrm{~mm}$, relative permittivity $\epsilon_{r}=11.7$, and relative permeability $\mu_{r}=1$ is used. The angular frequency is $\omega=2 \pi 10 \cdot 10^{9} \mathrm{~Hz}$. On the substrate a realistic structure for measuring the memory requirements and speed is placed. The metallization is shown in Fig. 9 and consists of a uniform antenna array. The size of the patches is $w=7.5 \mathrm{~mm}$ and the periodicity of the array is $T=\left(3 \lambda_{0} / 4\right)=22.5 \mathrm{~mm}$. Each individual patch is discretized using a nonuniform mesh. At the edges of each patch the grid is refined in order to model the edge current behavior more accurately. The number of unknowns $N$ is increased by adding more patches. The target accuracy is set at $\mathcal{E}=10^{-5}$. In Fig. 10 the CPU time needed for one iteration is plotted for a variable number of unknowns. Fig. 11 shows the memory requirements of the code. As predicted, with the new PML-MLFMA both the operation count and the memory require-

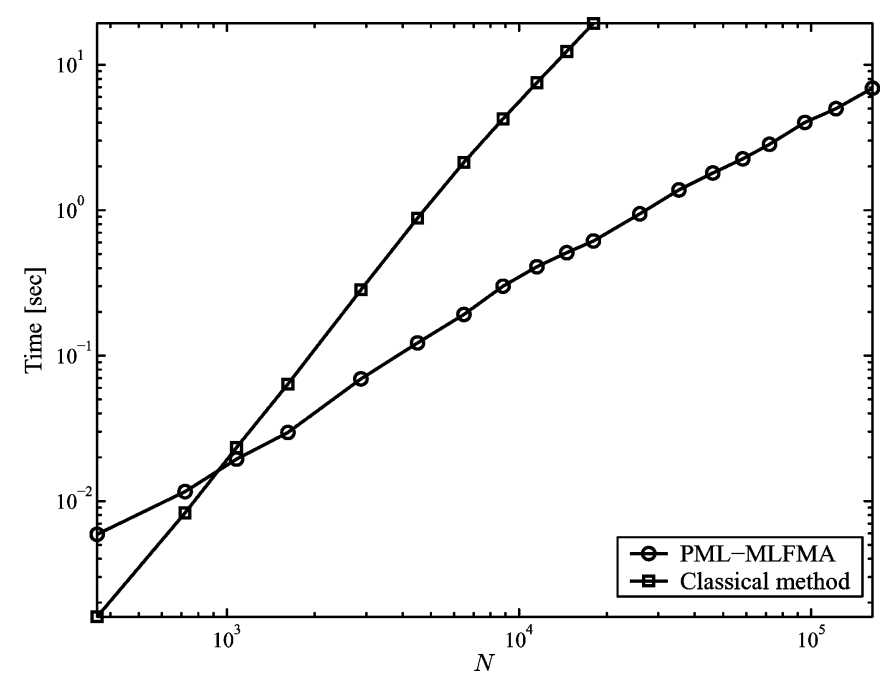

Fig. 10. CPU time for one matrix-vector multiplication.

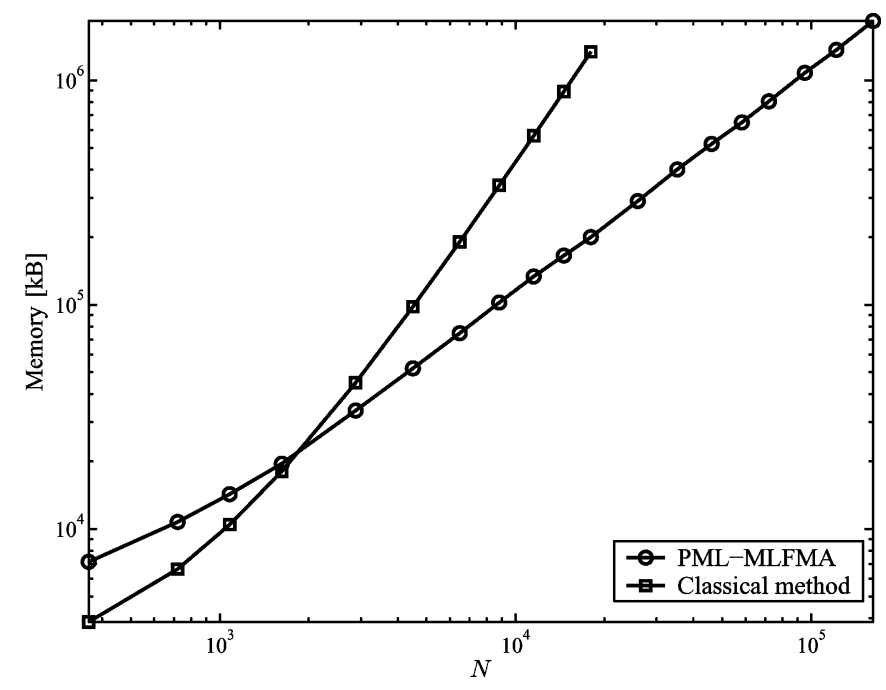

Fig. 11. Memory requirements.

ments scale as $O(N)$, as opposed to a classical method with an $O\left(N^{2}\right)$ complexity. Important to stress is that the cross-over point for the speed is found just below $N=1000$. Starting from about 2000 unknowns, there is also already a gain in memory efficiency. This is slightly larger than what is obtained for the speed and can be explained by the fact that even for small structures the MLFMA has a large fixed cost of memory, just for building the tree. These results are in line with those of free space FMMs and demonstrate that PML-MLFMA allows the modeling of very large planar structures.

\section{Application Examples}

This subsection presents computational results illustrating the applicability of the proposed scheme to the analysis of radiation and scattering by/from electrically large arrays. The far-field $\mathbf{E}^{\mathrm{s}, \mathrm{ff}}(R, \theta, \phi)$ scattered from an antenna array is expressed as

$$
\mathbf{E}^{\mathrm{s}, \mathrm{ff}}(R, \theta, \phi) \approx\left(\mathcal{F}_{\theta}(\theta, \phi) \hat{\boldsymbol{\theta}}+\mathcal{F}_{\phi}(\theta, \phi) \hat{\boldsymbol{\phi}}\right) \frac{e^{-j k_{0} R}}{R}
$$

for large $k_{0} R$, where $R=|\mathbf{r}|=\sqrt{x^{2}+y^{2}+z^{2}}$ and $\theta$ is the angle between $\mathbf{r}$ and the $z$-axis and $\phi$ the angle between the 


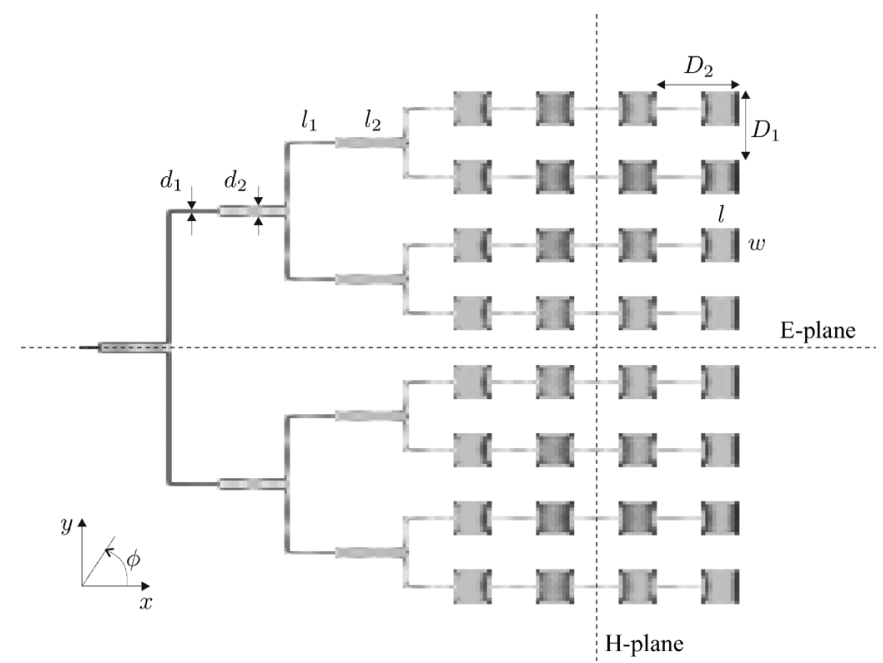

Fig. 12. Layout of the $8 \times 4$ microstrip array. The grayscale is an indication for the current density on the metallization.

$x$-axis and the projection of $\mathbf{r}$ onto the $x y$-plane. The radiation patterns $\mathcal{F}_{\theta}(\theta, \phi)$ and $\mathcal{F}_{\phi}(\theta, \phi)$ can be calculated from the currents on the metallization using a stationary phase method [34].

The first example involves the $8 \times 4$ microstrip array detailed in [44], [45] and shown in Fig. 12. The array is situated on a substrate with thickness $d=1.59 \mathrm{~mm}$, relative permittivity $\epsilon_{r}=2.2$, and relative permeability $\mu_{r}=1$. The various dimensions detailed in Fig. 12 are $l=10.08 \mathrm{~mm}, w=$ $11.79 \mathrm{~mm}, d_{1}=1.3 \mathrm{~mm}, d_{2}=3.93 \mathrm{~mm}, l_{1}=12.32 \mathrm{~mm}$, $l_{2}=18.48 \mathrm{~mm}, D_{1}=23.58 \mathrm{~mm}$, and $D_{2}=22.40 \mathrm{~mm}$. The array is fed by forcing a current at its input at angular frequency $\omega=2 \pi 9.42 \cdot 10^{9} \mathrm{~Hz}$. The array's radiation patterns in the E-plane $\left(\phi=0^{\circ}\right)$ and the H-plane $\left(\phi=90^{\circ}\right)$ are shown in Fig. 13(a) and (b) and compared with the results found in [45]. Very good agreement between both data sets is observed.

The second example involves the array first introduced in Section III-B (Fig. 9); the patch width is again $w=7.5 \mathrm{~mm}$ and the periodicity of the array, detailed in Fig. 9, is $T=3 \lambda_{0} / 4=$ $22.5 \mathrm{~mm}$. The structure is illuminated by a plane wave

$$
\begin{aligned}
\mathbf{E}^{\mathrm{PW}}(\mathbf{r}) & =\mathbf{E}_{0} e^{j\left(k_{x} x+k_{y} y+k_{z} z\right)} \\
& =\mathbf{E}_{0} e^{j k_{0}(\cos \phi \sin \theta x+\sin \phi \sin \theta y+\cos \theta z)}
\end{aligned}
$$

as indicated on Fig. 1. For this kind of excitation one can easily derive that at $z=d$ the incident field is of the following form:

$$
\check{\mathbf{E}}^{\mathrm{i}}(\boldsymbol{\rho})=\check{\mathbf{E}}_{0} e^{j\left(k_{x} x+k_{y} y+k_{z} d\right)}+\check{\mathbf{R}} e^{j\left(k_{x} x+k_{y} y-k_{z} d\right)}
$$

with $\check{\mathbf{R}}$ the transverse to $z$ strength of the wave reflected at the PEC-backed substrate. In our example again an angular frequency of $\omega=2 \pi 10 \cdot 10^{9} \mathrm{~Hz}$ is used and the plane wave has angles of incidence $\theta=30^{\circ}$ and $\phi=0^{\circ}$. The plane wave is linearly polarized along the $y$-axis and has a strength of $1(\mathrm{~V} / \mathrm{m})$, hence $\mathbf{E}_{0}=\hat{\mathbf{y}}$. For this illuminating plane wave and for the given microstrip substrate, the strength of the reflected wave is given by $\check{\mathbf{R}}=(0.195-j 0.981)(V / m) \hat{\mathbf{y}}$. In the $x z$-plane the scattering cross section $\left|\mathcal{F}_{\phi}(\theta) /\right| \mathbf{E}^{\mathrm{PW}}||^{2}$ is studied and a grating lobe in the radiation pattern at $\theta_{\mathrm{gr}}=56.4^{\circ}$ (apart from the specular reflection at $\theta_{\text {spec }}=-30^{\circ}$ ) is expected. For an infinite number of patches, one would obtain two discrete Dirac-like lobes at $\theta_{\mathrm{gr}}$ and $\theta_{\mathrm{spec}}$. Fig. 14 shows the scattering cross section

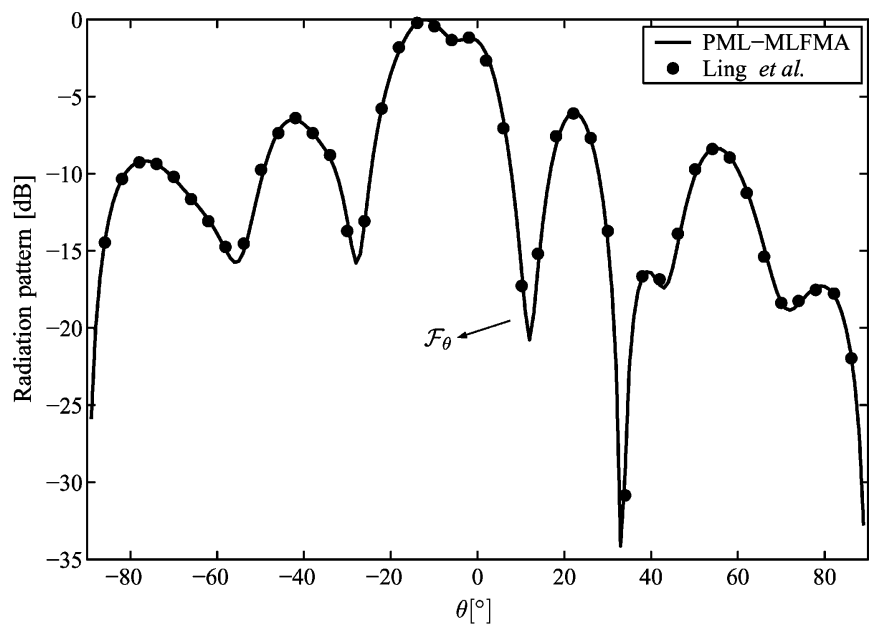

(a)

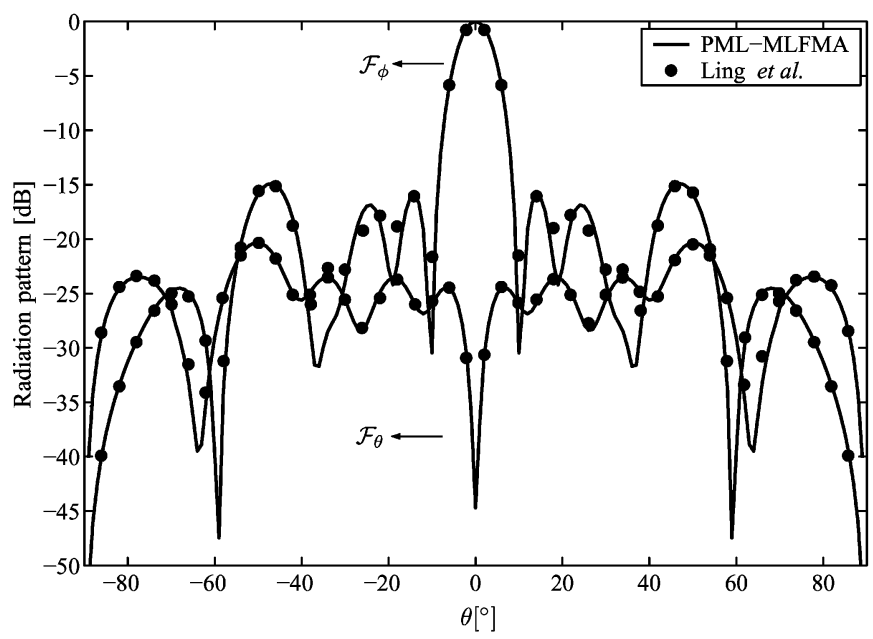

(b)

Fig. 13. Radiation patterns of the microstrip antenna array. (a) E-plane $(\phi=$ $\left.0^{\circ}\right)$ and (b) H-plane $\left(\phi=90^{\circ}\right)$.

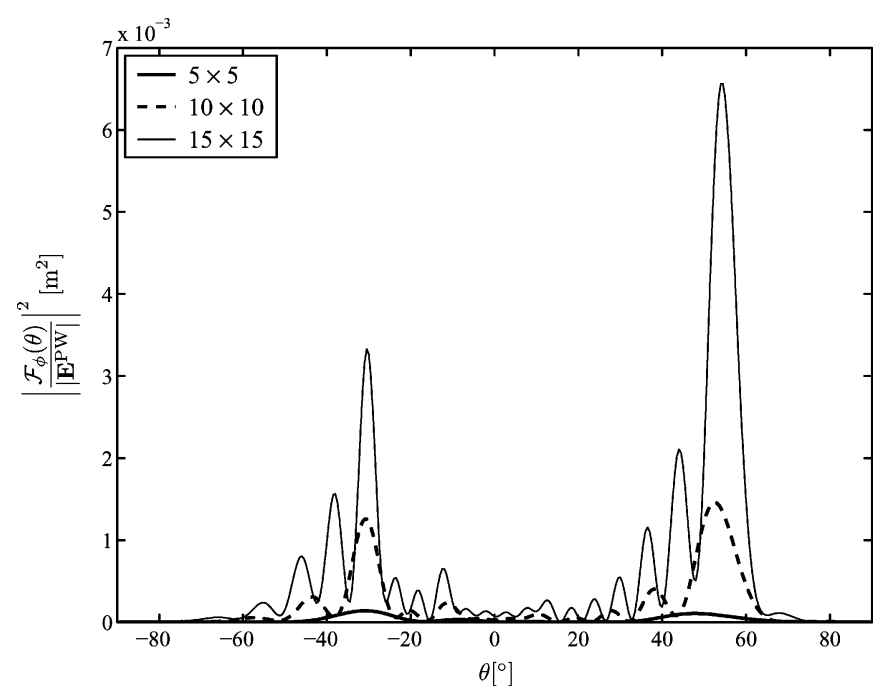

Fig. 14. Scattering cross section as a function of the number of patches.

in the $x z$-plane for a varying number of square patches. With an increasing number of patches, our result more and more resembles a pattern that only comprises two discrete lobes. The reader also notices the two predicted lobes at $\theta_{\mathrm{gr}}$ and $\theta_{\text {spec }}$. 


\section{CONCLUSION}

In this contribution, a novel MLFMA technique has been introduced to analyze radiation by and scattering from three-dimensional planar microwave structures. The method starts from a classical EFIE formulation solved with the MoM. The crux of our method resides in a recently developed new robust series expansion for the electric-electric Green dyadic $\overline{\mathbf{G}}_{e e}$ that is based on the application of PMLs. Each term in the series for $\overline{\mathbf{G}}_{e e}$ can be decomposed into a set of 2-D plane wave contributions. This allows a separation of the source and the observer in the Green function, leading to an MLFMA implementation. Only for near and for self-patch interactions, the PML-MLFMA formalism has to be abandoned and needs to be replaced by a classical technique. A new fast multiplication scheme has been implemented and a computational complexity of order $O(N)$ is achieved. The accuracy of the method has been extensively tested, which allows the reader to conclude that it is fully controllable. The proposed technique is applied to electrically large planar antenna arrays, clearly validating the accuracy and the capabilities of the method. The extension of the present PML-MLFMA technique to electrically very small planar structures using a low frequency version of the algorithm is currently under investigation.

\section{REFERENCES}

[1] R. F. Harrington, Field Computation by Moment Methods. Piscataway, NJ: IEEE Press, 1993.

[2] O. Axelsson, Iterative Solution Methods. New York: Cambridge Univ. Press, 1994

[3] C.-T. Tai, Dyadic Green Functions in Electromagnetic Theory, 2nd ed, ser. Series on electromagnetic waves. New York: IEEE Press, 1993.

[4] K. A. Michalski and J. R. Mosig, "Multilayered media Green's functions in integral equation formulations," IEEE Trans. Antennas Propag., vol. 45, no. 3, pp. 508-519, Mar. 1997.

[5] N. Faché, F. Olyslager, and D. De Zutter, Electromagnetic and Circuit Modeling of Multiconductor Transmission Lines. Oxford, U.K.: Oxford Univ. Press, 1993.

[6] F. Ling, C.-F. Wang, and J.-M. Jin, "Application of adaptive integral method to scattering and radiation analysis of arbitrarily shaped planar structures," J. Electromagn. Waves Applicat., vol. 12, no. 8, pp. 1021-1037, Aug. 1998

[7] J.-S. Zhao, W. C. Chew, C.-C. Lu, E. Michielssen, and J. Song, "Thinstratified medium fast-multipole algorithm for solving microstrip structures," IEEE Trans. Microwave Theory Tech., vol. 46, no. 4, pp. 395-403, Apr. 1998.

[8] B. Hu and W. C. Chew, "Fast inhomogeneous plane wave algorithm for electromagnetic solutions in layered medium structures: two-dimensional case," Radio Sci., vol. 35, no. 1, pp. 31-43, Jan.-Feb. 2000.

[9] _ - "Fast inhomogeneous plane wave algorithm for scattering from objects above the multilayered medium," IEEE Trans. Geosci. Remote Sensing, vol. 39, no. 5, pp. 1028-1038, May 2001.

[10] L. J. Jiang and W. C. Chew, "Low-frequency fast inhomogeneous planewave algorithm (LF-FIWPA)," Microwave Opt. Technol. Lett., vol. 40, no. 2, pp. 117-122, Jan. 2004.

[11] W. C. Chew, T. J. Cui, and J. M. Song, "A FAFFA-MLFMA algorithm for electromagnetic scattering," IEEE Trans. Antennas Propag., vol. 50, no. 11, pp. 1641-1649, Nov. 2002.

[12] J. P. Bérenger, "A perfectly matched layer for the absorption of electromagnetic waves," J. Comput. Phys., vol. 114, no. 2, pp. 185-200, Oct. 1994.

[13] W. C. Chew and W. H. Weedon, "A 3D perfectly matched medium from modified Maxwell's equations with stretched coordinates," Microwave Opt. Technol. Lett., vol. 7, no. 13, pp. 599-604, Sep. 1994.

[14] L. Knockaert and D. De Zutter, "On the stretching of Maxwell's equations in general orthogonal coordinate systems and the perfectly matched layer," Microwave Opt. Technol. Lett., vol. 24, no. 1, pp. 31-34, Jan. 2000

[15] H. Derudder, F. Olyslager, and D. De Zutter, "An efficient series expansion for the 2D Green's function of a microstrip substrate using perfectly matched layers," IEEE Microw. Guided Wave Lett., vol. 9, no. 12, pp. 505-507, Dec. 1999.
[16] F. Olyslager and H. Derudder, "Series representation of Green dyadics for layered media using PMLs," IEEE Trans. Antennas Propag., vol. 51, no. 9, pp. 2319-2326, Sep. 2003.

[17] F. Olyslager, "Discretization of continuous spectra based on perfectly matched layers," SIAM J. Appl. Math., vol. 64, no. 4, pp. 1408-1433, May 2004

[18] V. Rokhlin, "Rapid solution of integral equations of scattering theory in two dimensions," J. Comput. Phys., vol. 36, no. 2, pp. 414-439, Feb. 1990.

[19] R. Coifman, V. Rokhlin, and S. Wandzura, "The fast multipole method for the wave equation: a pedestrian prescription," IEEE Antennas Propag. Mag., vol. 35, no. 3, pp. 7-12, Jun. 1993.

[20] N. Engheta, W. D. Murphy, V. Rokhlin, and M. S. Vassiliou, "The fast multipole method (FMM) for electromagnetic scattering problems,' IEEE Trans. Antennas Propag., vol. 40, no. 6, pp. 634-641, Jun. 1992.

[21] K. A. Michalski and C. M. Butler, "Determination of current induced on a conducting strip embedded in a dielectric slab," Radio Sci., vol. 18, no. 6, pp. 1195-1206, Nov.-Dec. 1983.

[22] J. R. Mosig and F. E. Gardiol, "General integral-equation formulation for microstrip antennas and scatterers," Proc. Inst. Elect. Eng. Microwave Antennas Propag., pt. H, vol. 132, no. 7, pp. 424-432, Dec. 1985.

[23] J. L. Tsalamengas and J. G. Fikioris, "TM scattering by conducting strips right on the planar interface of a three-layered medium," IEEE Trans. Antennas Propag., vol. 41, no. 5, pp. 542-555, May 1993.

[24] J. L. Tsalamengas, "TE-scattering by conducting strips right on the planar interface of a three-layered medium," IEEE Trans. Antennas Propag., vol. 41, no. 12, pp. 1650-1658, Dec. 1993.

[25] F. Ling and J.-M. Jin, "Scattering and radiation analysis of microstrip antennas using discrete complex image method and reciprocity theorem," Microwave Opt. Technol. Lett., vol. 16, no. 4, pp. 212-216, Nov. 1997.

[26] D. Vande Ginste, H. Rogier, D. De Zutter, and F. Olyslager, "An efficient FMM-PML-MPIE formalism for 2D microstrips," in Proc. IEEE AP-S Int. Symp., vol. 1, Columbus, OH, Jul. 2003, pp. 31-34.

[27] _ _A fast multipole method for layered media based on the application of perfectly matched layers-the 2-D case," IEEE Trans. Antennas Propag., vol. 52, no. 10, pp. 2631-2640, Oct. 2004.

[28] B. Dembart and E. Yip, "A 3-D fast multipole method for electromagnetics with multiple levels," in Proc. 11th Annu. Rev. Progress Appl. Computat. Electromagn., Mar. 1995, pp. 621-628.

[29] M. A. Epton and B. Dembart, "Multipole translation theory for the three dimensional Laplace and Helmholtz equations," SIAM J. Sci. Comput., vol. 16, no. 4, pp. 865-897, Jul. 1995.

[30] J. M. Song and W. C. Chew, "Multilevel fast-multipole algorithm for solving combined field integral equations of electromagnetic scattering," Microwave Opt. Technol. Lett., vol. 10, no. 1, pp. 14-19, Sep. 1995.

[31] J. Song, C.-C. Lu, and W. C. Chew, "Multilevel fast multipole algortihm for electromagnetic scattering by large complex objects," IEEE Trans. Antennas Propag., vol. 45, no. 10, pp. 1488-1493, Oct. 1997.

[32] J. Sercu, N. Faché, F. Libbrecht, and P. Lagasse, "Mixed potential integral equation technique for hybrid microstrip-slotline multilayered circuits using a mixed rectangular-triangular mesh," IEEE Trans. Microwave Theory Tech., vol. 43, no. 5, pp. 1162-1172, May 1995.

[33] W. C. Chew, J.-M. Jin, E. Michielssen, and J. Song, Fast and Efficient Algorithms in Computational Electromagnetics. Boston, MA: Artech House, 2001

[34] L. B. Felsen and N. Marcuvitz, Radiation and Scattering of Waves. Piscataway, NJ: IEEE Press, 1994.

[35] F. Olyslager, Electromagnetic Waveguides and Transmission Lines Oxford, U.K.: Oxford Univ. Press, 1999.

[36] W. C. Chew, J. M. Jin, and E. Michielssen, "Complex coordinate system as a generalized absourbing boundary condition," in Proc. 13th Annu. Rev. Progress in Applied Electromagnetics, vol. 2, Monterey, CA, 1997, pp. 909-914.

[37] H. Derudder, F. Olyslager, D. De Zutter, and S. Van den Berghe, "Efficient mode-matching analysis of discontinuities in finite planar substrates using perfectly matched layers," IEEE Trans. Antennas Propag., vol. 49, no. 2, pp. 185-195, Feb. 2001.

[38] H. Rogier and D. De Zutter, "Bérenger and leaky modes in microstrip substrates terminated by a perfectly matched layer," IEEE Trans. Microwave Theory Tech., vol. 49, no. 4, pp. 712-715, Apr. 2001.

[39] I. V. Lindell, Methods for Electromagnetic Field Analysis. Oxford, U.K.: Oxford Univ. Press, 1992.

[40] L. F. Knockaert and D. De Zutter, "On the completeness of eigenmodes in a parallel plate waveguide with a perfectly matched layer termination," IEEE Trans. Antennas Propag., vol. 50, no. 11, pp. 1650-1653, Nov. 2002

[41] F. Olyslager, Mathematical Modeling of Wave Phenomena, B. Nilsson, Ed. Växjö, Sweden: Växjö Univ. Press, 2003, ch. Series approximation for Green functions. 
[42] H. Rogier and D. De Zutter, "Convergence behavior and acceleration of the Berenger and leaky modes series composing the 2-D Green's function for the microstrip substrate," IEEE Trans. Microwave Theory Tech., vol. 50, no. 7, pp. 1696-1704, Jul. 2002.

[43] W. C. Chew, H. Y. Chao, T. J. Cui, C. C. Lu, S. Ohnuki, Y. C. Pan, J. M Song, S. Velamparambil, and J. S. Zhao, "Fast integral equation solvers in computational electromagnetics of complex structures," Eng. Analysis With Boundary Elements, vol. 27, no. 8, pp. 803-823, Sep. 2003.

[44] C.-F. Wang, F. Ling, and J.-M. Jin, "A fast full-wave analysis of scattering and radiation from large finite arrays of microstrip antennas," IEEE Trans. Antennas Propag., vol. 46, no. 10, pp. 1467-1474, Oct. 1998.

[45] F. Ling, C.-F. Wang, and J.-M. Jin, “An efficient algorithm for analyzing large-scale microstrip structures using adaptive integral method combined with discrete complex-image method," IEEE Trans. Microwave Theory Tech., vol. 48, no. 5, pp. 832-839, May 2000.

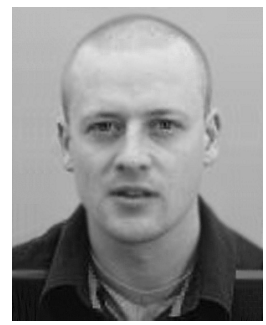

Dries Vande Ginste was born in 1977. He received the electrical engineering and Ph.D. degrees, from Ghent University, Gent, Belgium, in 2000 and 2005, respectively.

Currently, he is with the Department of Information Technology (INTEC), Ghent University, as a Postdoctoral Researcher. His main interest is in the area of fast techniques for the modeling of layered media.

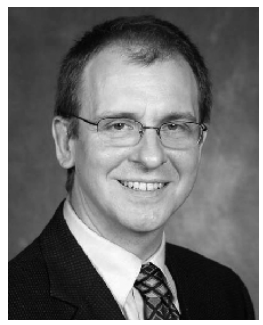

Eric Michielssen (M'95-SM'99-F'02) received the M.S. degree in electrical engineering (summa cum laude) from the Katholieke Universiteit Leuven (KUL), Belgium, in 1987 and the Ph.D. degree in electrical engineering from the University of Illinois at Urbana-Champaign (UIUC), in 1992.

He was a Research and Teaching Assistant in the Microwaves and Lasers Laboratory at KUL and the Electromagnetic Communication Laboratory at UIUC from 1987 to 1988 and 1988 to 1992, respectively. He joined the Faculty of the Department of Electrical and Computer Engineering at the University of Illinois as a Visiting Assistant Professor in 1992, became Assistant Professor of electrical and computer engineering in 1993, and became an Associate Professor in 1998. Since 1995 he has been Associate Director of the Center for Computational Electromagnetics at UIUC. In 2005, he joined the University of Michigan as Professor of Electrical Engineering and Computer Science. He is the Beckman Fellow in the Center for Advanced Studies. He authored or coauthored over 130 journal papers and book chapters and over 190 in conference proceedings. His research interests include all aspects of theoretical and applied computational electromagnetics. His principal research focus has been on the development of fast frequency and time domain integral-equation-based techniques for analyzing electromagnetic phenomena, and the development of robust optimizers for the synthesis of electromagnetic/optical devices. He was an Associate Editor for Radio Science.

Prof. Michielssen is a Member of International Union of Radio Scientists (URSI) Commission B. He received a Belgian American Educational Foundation Fellowship in 1988 and a Schlumberger Fellowship in 1990. He received a 1994 URSI Young Scientist Fellowship, a 1995 National Science Foundation CAREER Award, and the 1998 Applied Computational Electromagnetics Society (ACES) Valued Service Award. In addition, he was named 1999 URSI-United States National Committee Henry G. Booker Fellow and was selected as the recipient of the 1999 URSI Koga Gold Medal. He received UIUC's 2001 Xerox Award for Faculty Research, was appointed 2002 Beckman Fellow in the UIUC Center for Advanced Studies, named 2003 Scholar in the Tel Aviv University Sackler Center for Advanced Studies, and selected as the UIUC 2003 University Scholar and Sony Scholar. He was Technical Chairman of the 1997 ACES Symposium (Review of Progress in Applied Computational Electromagnetics, March 1997, Monterrey, CA), and from 1998 to 2001 and 2002 to 2003, served on the ACES Board of Directors and as ACES Vice-President from 1998 to 2001. From 1998 to 2005, he served as Associate Editor for the IEEE TRANSACTIONS ON ANTENNAS AND PROPAGATION.

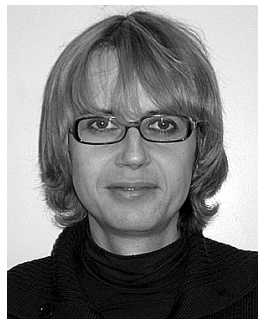

Femke Olyslager (S'90-M'94-SM'99-F'05) was born in 1966. She received the Electrical Engineering degree in 1989 and the Ph.D. degree in 1993, both from Ghent University, Gent, Belgium.

At present she is a Full Professor in electromagnetics at Ghent University. Her research concerns different aspects of theoretical and numerical electromagnetics. She authored or coauthored more than 200 papers in journals and proceedings. She coauthored Electromagnetic and Circuit Modeling of Multiconductor Transmission Lines (Oxford, U.K.: Oxford University Press, 1993) and authored Electromagnetic Waveguides and Transmission Lines (Oxford, U.K.: Oxford University Press, 1999).

Dr. Olyslager is Assistant Secretary General of International Union of Radio Scientists (URSI), an Associate Editor of the IEEE TRANSACTIONS ON ANTENNAS AND PROPAGATION and was Associate Editor of Radio Science. In 1994 she became laureate of the Royal Academy of Sciences, Literature and Fine Arts of Belgium. She received the 1995 IEEE Microwave Prize for the best paper published in the 1993 IEEE TRANSACTIONS ON MiCROWAVE THEORY AND TECHNIQUES and the 2000 Best Transactions Paper award for the best paper published in the 1999 IEEE TRANSACTIONS ON ELECTROMAGNETIC COMPATIBILITY. In 2002 she received the Issac Koga Gold Medal at the URSI General Assembly and in 2004 she became laureate of the Royal Flemish Academy of Belgium.

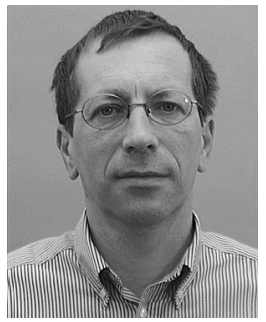

Daniël De Zutter (M'92-SM'96-F'00) was born in 1953. He received the M.Sc. degree in electrical engineering, the $\mathrm{Ph} . \mathrm{D}$. degree, and completed a thesis leading to a degree equivalent to the French Aggrégation or the German Habilitation, all from the University of Ghent, Gent, Belgium, in 1976, 1981, and 1984, respectively.

From 1976 to 1984, he was a Research and Teaching Assistant at Ghent University, where he is now a Full Professor of electromagnetics in the Department of Information Technology and Dean of the Faculty of Engineering. From 1984 to 1996, he was with the National Fund for Scientific Research of Belgium. As author or coauthor he has contributed to more than 135 international journal papers and 140 papers in conference proceedings. In 1993, he coauthored Electromagnetic and Circuit Modeling of Multiconductor Transmission Lines, Oxford Engineering Science Series (Oxford, U.K.: Oxford Univ. Press, 1993). Most of his earlier scientific work dealt with the electrodynamics of moving media. His research now focusses on all aspects of circuit and electromagnetic modeling of high-speed and high-frequency interconnections and packaging, on electromagnetic compatibility (EMC), and numerical solutions of Maxwell's equations.

Dr. De Zutter received the 1990 Montefiore Prize of the University of Lige and was the co-recipient the 1995 IEEE Microwave Prize Award from the IEEE Microwave Theory and Techniques Society for Best Publication in the Field of Microwaves for the Year 1993. In 1999 he received the Transactions Prize Paper Award from the IEEE EMC Society. In 2000 he was elected to the grade of Fellow of the IEEE “for the application of Maxwell's equations and for the development of numerical solution methods in electromagnetic scattering, antennas and microwave circuits." 